\title{
An Improved Neural Network Algorithm for Remote Sensing Image Classification
}

\author{
Liang Zhao \\ Department of Basic, Jilin Justice Officer Academy, Jilin, Changchun130062, China \\ Received: November 8, 2020. Revised: December 18, 2020. Accepted: December 21, 2020. \\ Published: December 22, 2020.
}

\begin{abstract}
The appearance of hyperspectral remote sensing image further improves the accuracy of remote sensing image classification, but the data of hyperspectral remote sensing image is large, and the processing hyperspectral remote sensing image has high complexity and low efficiency. A remote sensing image classification algorithm based on improved bilinear recurrent neural network (BLRNN) model is proposed, this paper gives the definition of bilinear recurrent neural Network and the description of network structure, and optimizes and improves the bilinear recurrent neural network from two aspects of network structure and pruning process, and uses the genetic algorithm global search to trim. Compared with the original feature, PCA and BPNN algorithm, the results show that the BLRNN algorithm has been greatly improved in classification accuracy and classification time, and the image processing efficiency is improved.
\end{abstract}

Keywords-remote sensing image classification, feature extraction, classification accuracy, neural network, structure optimization

\section{INTRODUCTION}

$\mathrm{W}$ ITH the rapid development of remote sensing technology, a large amount of remote sensing image database appears, which brings difficulties for remote sensing image management and query[1-2]. Therefore, the construction of high efficiency and high precision remote sensing image classification algorithm has become a hotspot in the field of remote sensing image research. The classification of remote sensing images is inseparable from the extraction of professional information, the prediction of dynamic change, the production of thematic maps and the establishment of remote sensing databases. How to classify remote sensing images efficiently and accurately becomes an important research content in this field, which belongs to the research category of pattern recognition [3-5].

At present, image classification methods can be divided into supervised method and unsupervised method according to whether prior knowledge is used for classification. Among them, the supervision classification method is most noticeable. The supervised classification is a kind of statistical decision classification method with high accuracy, and the discriminant function and the corresponding criterion are determined by the prior knowledge of the class and category of the known samples. According to the function and criterion, the pixels in the image are classified into each given class and the image classification is completed. The commonly used supervised classification methods are maximum likelihood (ML) classification, and nonparametric classification methods include $\mathrm{k}$ nearest neighbor classification algorithm (KNN), $\mathrm{K}$ mean value algorithm (K-means), Fuzzy Classification, classification method based on Fisher Criterion, SVM classification method, the classification method based on Markov model and so on [6-10]. In these methods, ML algorithm is a widely used image classification method, and most of the image can be more accurate classification results with fast and simple characteristics.

The remote sensing image algorithm based on pattern recognition mainly includes the collection and preprocessing of remote sensing images, the feature extraction and selection of remote sensing images, the construction of remote sensing image classifier, and the key steps are: feature extraction, filter and classifier design. Since remote sensing images have many characteristics such as more bands and large amount of data, the original features of remote sensing images are huge, and if the original remote sensing images are used directly for classification, the Remote Sensing image classification is huge, the classification is time-consuming, and the real-time character of remote sensing image classification is affected. In order to solve the characteristics of remote sensing image features, the principal Component Analysis feature reduction method can be used to reduce the number of features by the principle of feature 
transformation, to select Representative features to describe the original features, and to reduce the number of features while preserving the physical meaning of the original features. But the principal component analysis method is easy to destroy the spatial structure of the original features of remote sensing images, and the features of the selection are weaker.

Since the 1990s, with the rapid development of pattern recognition and artificial intelligence theory, the theory of Artificial neural network is becoming more and more mature, and the algorithm is becoming more and more perfect, and it has achieved better results in the classification of remote sensing images[11-12]. However, due to the lack of sufficient theoretical basis for the selection of network structure and parameters, it is easy to fall into local minima and so on, which depends on the empirical factors. Therefore, the problem of "dimensionality disaster" is easy to appear, and the probability of the classification result of remote sensing image near "over fit" is higher, while the support vector machine does not appear "dimensionality disaster", the Remote sensing image classification time is longer and can not meet the classification requirement of the mass remote sensing image data [13].

To address the above problems, a remote sensing image classification algorithm based on improved bilinear recurrent neural network (BLRNN) is proposed in this paper, the definition of bilinear recurrent neural Network and the description of network structure are presented, and the double linear recurrent neural network is improved from two aspects of network structure and pruning process respectively. The genetic algorithm is used to trim the global search.

\section{MethdologY}

In 1994, Park proposed a bilinear recurrent neural network (bilinear recurrent neural network, BLRNN), which solves the limitations of the RNN's inability to use linear inputs and the ability to model nonlinear modeling, compared to other higherorder neural networks, BLRNN can quickly approximate any nonlinear dynamic system [14]. Bilinear polynomial neural Network is a simple periodic nonlinear system model, for a onedimensional input/output system, its input and output relation is given by formula (1):

$$
y[n]=\sum_{i=0}^{N-1} \sum_{j=1}^{N-1} b_{i j} y[n-j] x[n-i]+\sum_{i=1}^{N-1} a_{i} y[n-i]+\sum_{i=0}^{N-1} c_{i} x[n-i]
$$

where $x[i]$ is input, $y[i]$ is output, $N$ is the order of neural network recursion. In order to be general, we set up a bilinear recurrent neural network which contains $N$ input, $M$ hidden layer and K-order polynomial. In such cases, the BLRNN input is shown in the formula (2), and the structure of the BLRNN is shown in the formula (3).

$$
\begin{gathered}
X[n]=\left[x_{1}[n], x_{2}[n], \cdots, x_{N}[n]\right]^{T} \\
\operatorname{net}_{p}[n]=w_{p}+A_{p}^{T} Z[n]+Z[n]^{T} B_{p} X[x]+C_{p}^{T} X[n]
\end{gathered}
$$

where, $w_{p}$ is the weight of the bias neuron, a is the weight vector of the recursive part, $B$ is the double linear part and the weight matrix, $C$ is the weight vector of the feedforward part. The output of the BLRNN can be represented by a formula (4):

$$
o_{p}[n]=\phi\left(n e t_{p}[n]\right)
$$

Where $\phi$ is the activation function of the hidden layer. Fig. 1 shows a simple bilinear neural network architecture diagram, which can be described by type

$$
\begin{aligned}
& s(n)=\sum_{m=1}^{p} w_{m}(n) u(n-m+1)+\sum_{r=1}^{2} b_{r}(n) u(n-r) \\
& +\sum_{r=1}^{2} \sum_{m=1}^{p} c_{m}(n) u(n-m+1) a(n-r)
\end{aligned}
$$

where $x(n)$ and $S(n)$ are BLRNN inputs and outputs, the $w_{m}$ is the network connection weight value, the $b_{r}$ is the connection weight of the feedback unit, and the $c_{r m}$ is the connection weight value of the linear feedback unit.

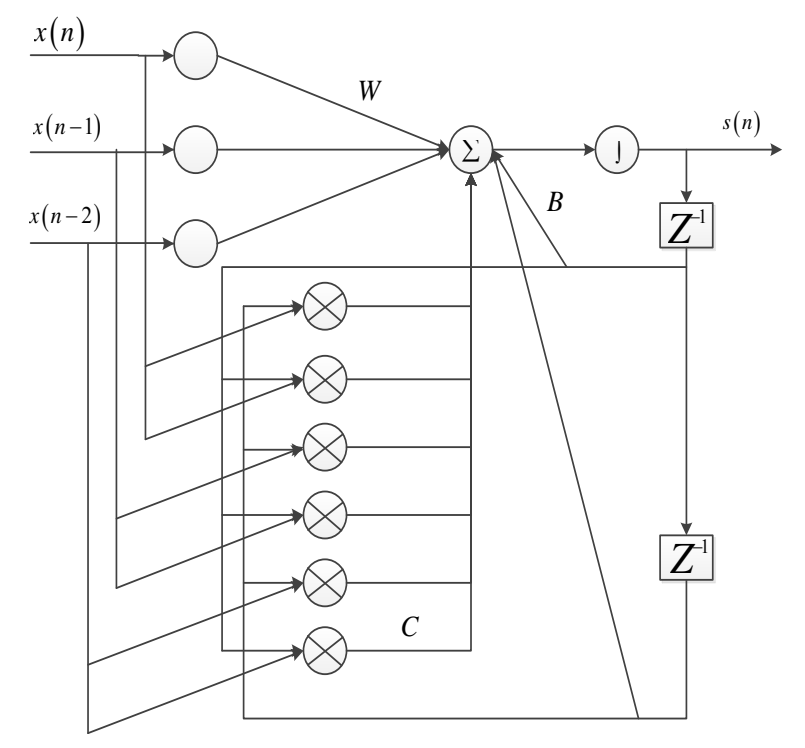

Fig. 1 The structure of double linear recurrent neural network

\section{A. BLRNN Neural Network Optimization}

Although bilinear neural network has been widely used in many fields, its computational capacity is still a bottleneck of 
restricting the generalization of this method. In this paper, the BLRNN is optimized from two aspects of the structure simplification and pruning algorithm, and the optimized bilinear neural network is called OBLRNN (optimized blrnn), which eliminates the unnecessary neuron or connection weights in the calculation process, thus reducing the whole computational amount and improving the efficiency.

The structure of the BLRNN is $Q_{\mathrm{i}}-Q_{\mathrm{h}}-Q_{\mathrm{o}}$, the feedback tap is $Q_{\mathrm{f}}$, in order to facilitate the analysis, this set $Q_{0}=1$, then the corresponding multiplication calculation is:

$$
Q_{c}^{\prime}=Q_{f} Q_{i}+\left(2 Q_{i}+Q_{f}\right) Q_{h}+Q_{h}
$$

In fact, the feedforward units of bilinear neural networks are not fully used, but exist redundancy, on the one hand, in the actual problem, the sample size is often very large, if fully used, will cause too large network size, on the other hand, will introduce too many parameters. Therefore, without affecting the BLRNN performance, it is possible to reduce the number of feed-forward input units, so as to achieve the purpose of simplifying the structure. After the method is introduced, the formula (5) can be overridden as shown in the following style:

$$
\begin{aligned}
& s_{p}^{\prime}[n]=w_{p}+\sum_{k_{1}=0}^{N_{1}-1} a_{p k_{1}} o_{p}\left[n-k_{1}\right]+\sum_{k_{2}=0}^{N_{1}-1} c_{p k_{2}}\left[n-k_{2}\right]+ \\
& \sum_{k_{1}=0}^{N_{1}-1} \sum_{k_{2}=S}^{E} b_{p k_{k} k_{2}} o_{p}\left[n-k_{1}\right]\left[n-k_{2}\right]
\end{aligned}
$$

Where, $E=\frac{Q_{i}-1}{2}+p, \quad S=\frac{Q_{i}-1}{2}-p$. The multiplication of the formula after simplification is as follows

$$
Q_{c}=\left(Q_{i}+Q_{f}+Q_{f} Q_{i}\right) Q_{h}+Q_{h}
$$

Another formula (6) minus the formula (8), can be obtained after the optimization structure of the multiplier number as shown in the formula (9), you can see that the degree of simplification and blrnn input layer, hidden layer and the number of feedback taps.

$$
\Delta Q=Q_{h} Q_{f} Q_{i}-\left(Q_{h}+Q_{f}\right) Q_{i}
$$

\section{B. Pruning Algorithm}

In order to reduce the computation of BLRNN, the pruning algorithm is used to optimize the process and remove as many redundant weights and neurons as possible. Many pruning methods are summarized, and the method of pruning based on genetic algorithm (GA) has excellent performance. Compared with other pruning methods, genetic algorithms only use less hypothetical conditions to a large extent to ensure the integrity of the original method. In addition, the pruning process based on genetic algorithm has good convergence, with less computation time and high robustness, and is an effective tool for global search method with its flexibility and robustness. Figure 2 shows a flowchart for pruning the BLRNN genetic algorithm, with a total of 3 caveats.

(1) Encoding method: Each weight is encoded by a binary number ("0" or "1"). 1 "means the connection is valid," 0 "means the connection does not exist, when all input weights of a node or all output weights are" 0 ", then the node can be deleted. The entire network is a sequence of chromosomes consisting of " 1 " and " 0 ", which is equal to the number of heavy ownership in the network.

(2) Fitness function: The purpose of structural optimization is to on the premise of ensuring basic performance, as much as possible to streamline BLRNN structure, in order to achieve this, the number of hidden layers is set to 1 , at this time the corresponding fitness function as shown in the formula (10), where $Q_{\mathrm{i}}, Q_{\mathrm{o}}$ and $Q_{\mathrm{f}}$ are the number of input layer neurons,

The number of output layer neurons and the number of feedback taps, $C_{\mathrm{i}}, C_{\mathrm{o}}$ and $C_{\mathrm{f}}$ are corresponding adjustment coefficients respectively.

$$
f(\text { net })=\frac{1}{1+C_{i} Q_{i}+C_{f} Q_{f}+C_{o} Q_{o}}
$$

(3) Evolution rules: According to the operating rules of the fittest, through the roulette selection method evolution, the highly adaptable individuals are inherited to the next generation group of probability, low fitness of individuals, the probability of heredity to the next generation group is small. In the mutation process, when a neuron is removed from the hidden layer or input layer, the corresponding ownership weight of the neuron is also deleted. 


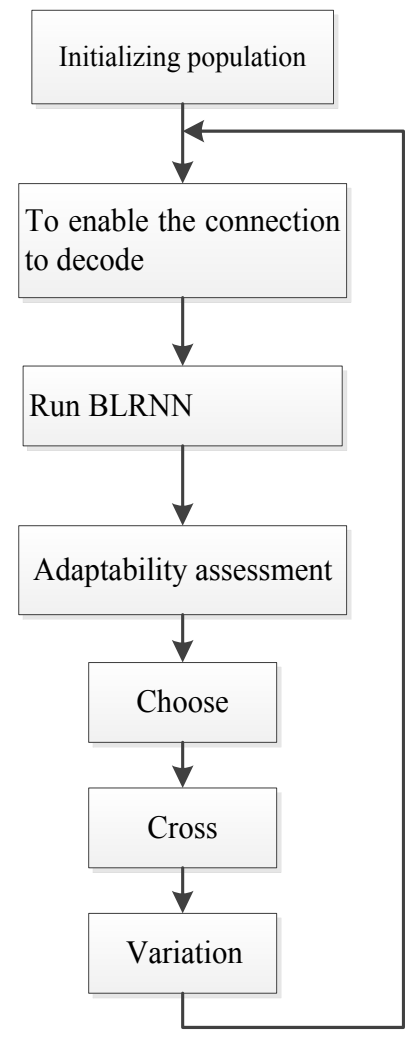

Fig.2 Flowchart of pruning optimization algorithm

\section{EXPERIMENTS AND ANALYSIS}

\section{A. Test sample Processing}

In order to verify the classification effect of remote sensing image based on quantum particle swarm, we choose MATLAB as the Simulation tool, select the United States Land Use classification dataset ucm_landuse_21, it contains 21 kinds of land use scene types, each type has $256 \times 256 \times 3$ size 100 scene chart. The remote sensing image classification process is shown in Figure 3. The 5 types of remote sensing images were selected as test subjects, such as rivers, runways, forest, agricultural, buildings, the number of training samples and the number of test samples as shown in table 1 . The scene of remote sensing image is classified by its function definition, that is to say, there are many types of land cover in an image, so the classification is more difficult than the general scene classification.

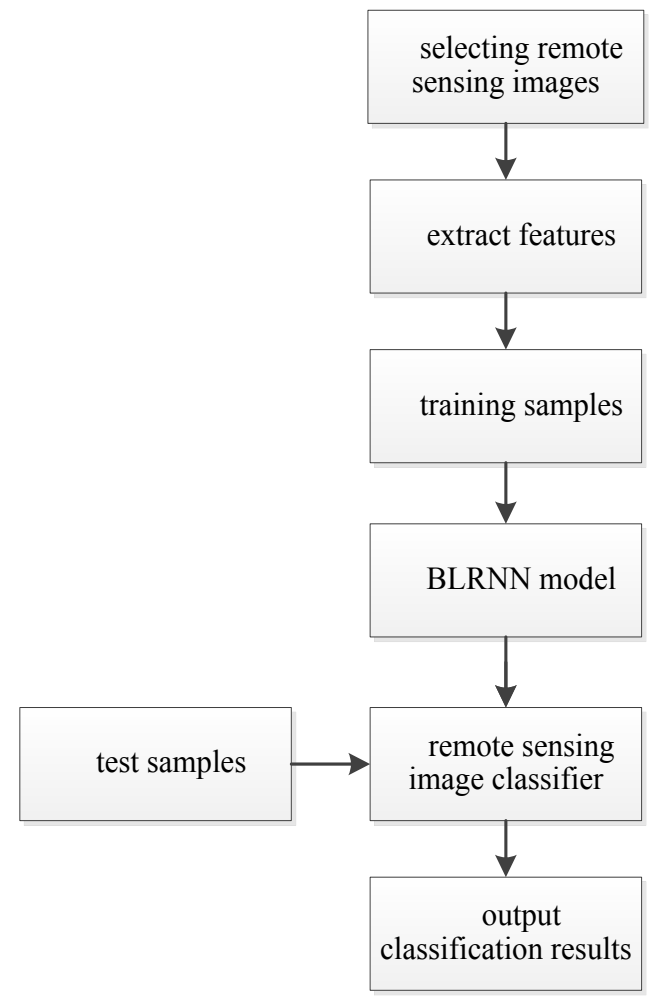

Fig.3 Process of remote sensing image classification by BLRNN

Firstly, the remote sensing image is treated with gray scale, and the experimental results are shown in Figure 4 (b).

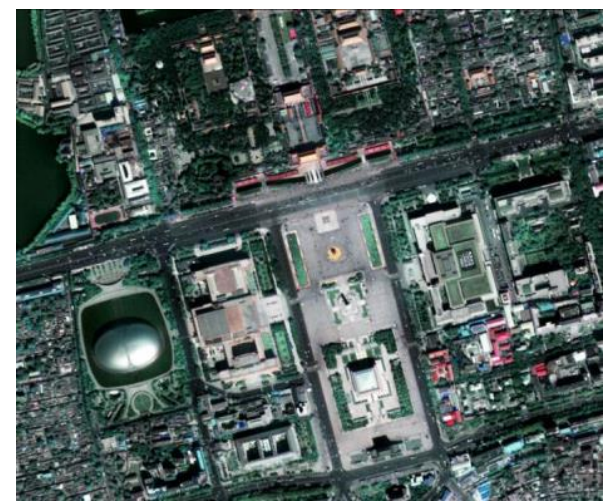

(a) Original graph 


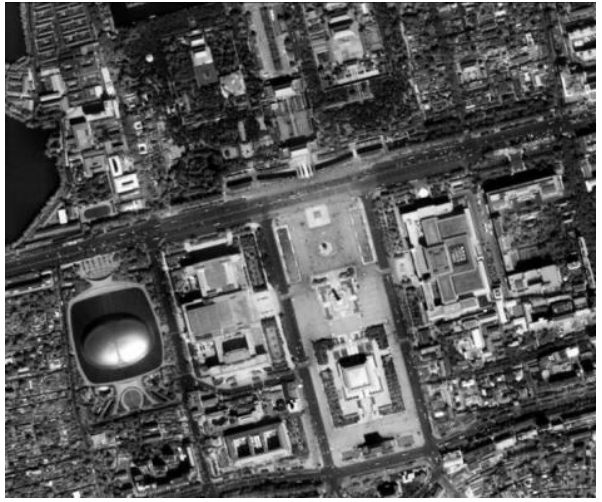

(b) Grayscale graph

Fig. 4 Remote sensing image

Table 1 The amount of training and test sample

\begin{tabular}{|l|l|l|}
\hline Types & Training samples & Test samples \\
\hline Rivers & $20-300$ & 2358 \\
\hline Runways & $20-300$ & 2185 \\
\hline Forest & $20-300$ & 5820 \\
\hline Agricultural & $20-300$ & 3512 \\
\hline Buildings & $20-300$ & 3208 \\
\hline
\end{tabular}

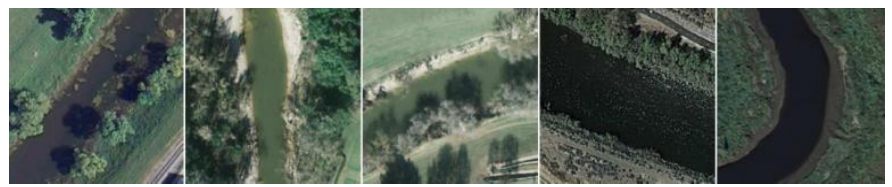

(a) Rivers

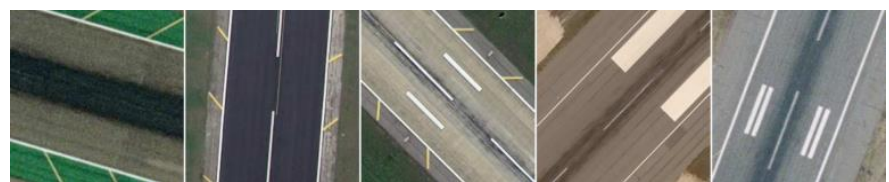

(b) Runways

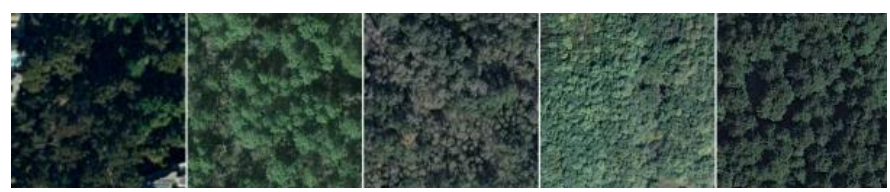

(c) Forest

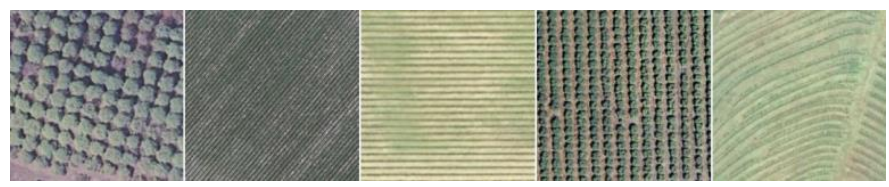

(d) Agricultural

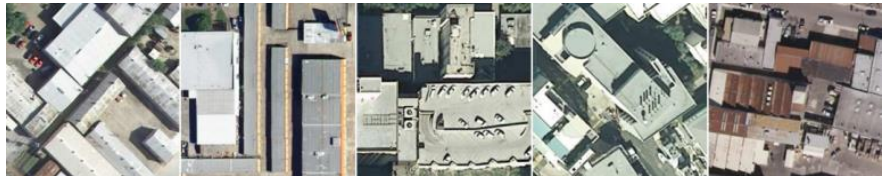

(e) Buildings

Fig. 5 The ground truth dataset, each of 21 land-use classes. Five samples from each class are shown above.

\section{B. Experimental Analysis}

Firstly, it verifies the superiority of OBLRNN and other feature selection algorithms, selects the original feature method (OFD), PCA and OBLRNN algorithms to carry on the contrast experiment, each algorithm optimizes the original remote sensing image feature, obtains the different quantity characteristic, then uses the $\mathrm{KNN}$ algorithm to design the remote sensing image classifier, All algorithms of remote sensing image classification accuracy and classification time as shown in Figure 6 and Figure 7, respectively.

1) The classification accuracy of remote sensing image of all feature screening algorithms is superior to that of the original feature, which shows that there are a lot of characters in the original remote sensing image which have negative effect on the classification result of remote sensing image, and can remove the negative features through feature selection, so as to effectively improve the classification result of remote. Compared with the OFD method, the classification accuracy of PCA algorithm is higher than that of $5.1 \%$, OBLRNN algorithm, the average precision is $8.1 \%$ and $2.8 \%$ respectively compared with OFD method and PCA algorithm.

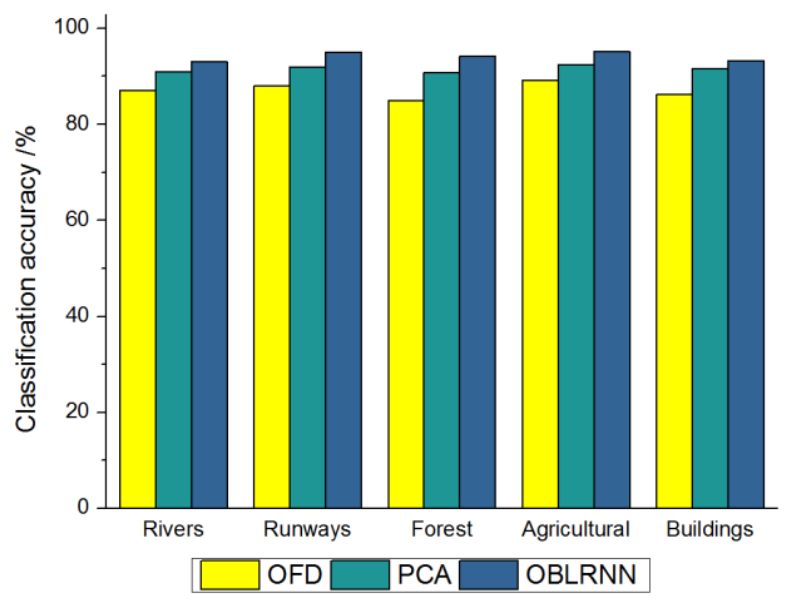

Fig. 6 Comparisons of classification accuracy of remote sensing images with different feature selection algorithms 


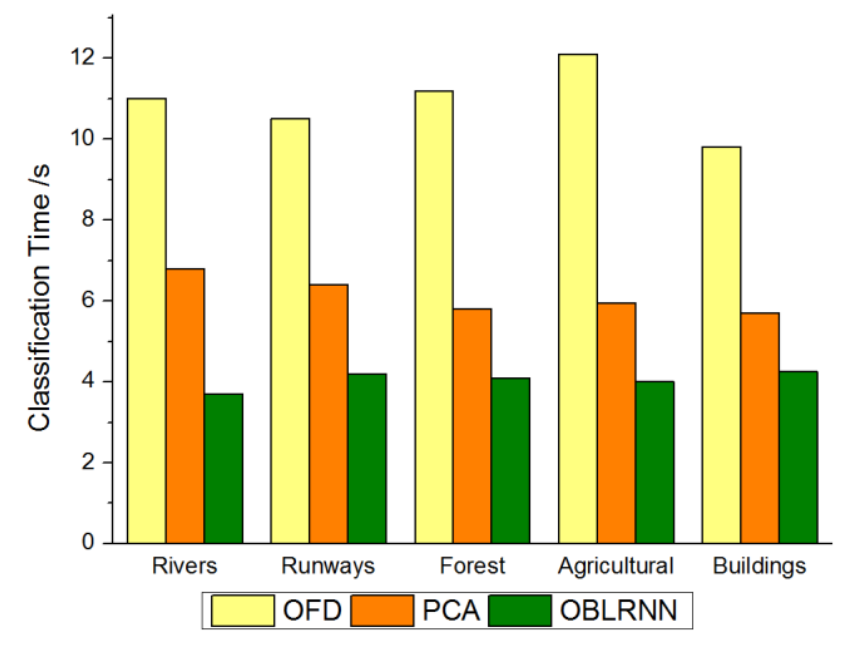

Fig. 7 Comparisons of classification time of remote sensing images with different feature selection algorithms

2) Compared to other remote sensing image feature screening algorithms, OBLRNN remote sensing Image classification accuracy is the highest, which is mainly OBLRNN can better select the best classification features of remote sensing images, and obtain more ideal classification results of remote sensing images.

3 ) The original feature of remote sensing image classification time is the longest, this is mainly because of the number of original features, resulting in remote sensing image classification of the structure of more complex, more computational volume. The classification structure of remote sensing image can be simplified by feature screening, and the learning speed of remote sensing image classifier is accelerated, which is suitable for the classification of complex remote sensing images, especially the advantages of OBLRNN, and the shortest classification time of remote sensing images. Compared with the OFD method, the classification accuracy of PCA algorithm is less than that of $22 \%$, OBLRNN algorithm and OFD method and PCA algorithm, the classification time decreases $33 \%$ and $25 \%$ respectively.

\section{CONCLUSION}

In order to solve the problem of complex data types in hyperspectral remote sensing classification, poor classification results and low classification efficiency, this paper proposes a remote sensing image classification algorithm based on structural optimization recurrent neural network, which is aimed at the disadvantages of large computational capacity and slow convergence speed of BLRNN algorithm. In this paper, we optimize the network structure and pruning algorithm, and introduce the genetic algorithm to solve the global optimal solution. The above 2 aspects of improvement and complementarity, can quickly and effectively find the best BLRNN classifier parameters, improve BLRNN classification accuracy, through the United States Land use classification data set UCM_LANDUSE_21 classification of the experiment, BLRNN algorithm in classification accuracy and classification time has been greatly improved, the improved BLRNN algorithm has high feasibility. Future research will focus on how to further optimize the structure and parameters of the remote sensing image classification method, improve the accuracy of classification and reduce the running time of the algorithm.

\section{REFERENCES}

[1] C.H. Chen, P.-G. "Peter Ho, Statistical pattern recognition in remote sensing", Pattern Recognition, vol.41, no.9, pp.2731-2741, 2008.

[2] P. Soille, M. Pesaresi. "Advances in mathematical morphology applied to geoscience and remote sensing", IEEE Transactions on Geoscience and Remote Sensing, vol.40, no.9, 2042-2055, 2002.

[3] P. Monasse, F. Guichard. "Fast Computation of a contrast-invariant image representation", IEEE Transactions on Image Processing, vol.9, no.5, pp.860-872, 2000.

[4] J. Debayle, J.-C. Pinoli. "General adaptive neighborhood image processing - part II", Journal of Mathematical Imaging and Vision, vol.25, no.2, pp.267-284, 2006.

[5] J. Chanussot, J.A. Benediktsson, M. Fauvel. "Classification of remote sensing images from urban areas using a fuzzy possibilistic model", IEEE Geoscience Remote Sensing Letters, vol.3, no.1, pp.40-44, 2006.

[6] Moore, M.M., Bauer, M.E. "Classification of forest vegetation in NorthCentral Minnesota using Landsat multispectral scanner and thematic mapper data", Forest Science, vol.36, no.2, pp.330-342, 1990.

[7] Kim, S., McGaughey, R.J., Andersen, H.-E. et al., "Tree species differentiation using intensity data derived from leaf-on and leaf-off airborne laser scanner data", Remote Sensing of Environment, vol.13, no.8, pp.1575-1586, 2009.

[8] Kim, S.-R., Lee, W.-K., Kwak, D.-A., et al. "Forest cover classification by optimal segmentation of high resolution satellite imagery", Sensors, vol.11, pp.1943-1958, 2011.

[9] Mallinis, G., Koutsias, N., Tsakiri-Strati, M., et al. 2008. "Object-based classification using Quickbird imagery for delineating forest vegetation polygons in a Mediterranean test site", Isprs Journal of Photogrammetry \& Remote Sensing, vol.63, no.2, pp.237-250, 2008.

[10] Carleer, A., Wolff, E., 2004. "Exploitation of very high resolution satellite data for tree species identification", Photogrammetric Engineering \& Remote Sensing, vol.70, no.1, pp.135-140, 2004.

[11] Packalén, P., Suvanto, A., Maltamo, M., "A two stage method to estimate species-specific growing stock", Photogrammetric Engineering \& Remote Sensing, vol.75, no.12, pp.1451-1460, 2009.

[12] Stavrakoudis, D.G., Dragozi, E., Gitas, I.Z.,et al. "Decision fusion based on hyperspectral and multispectral satellite imagery for accurate forest species mapping", Remote Sensing, vol.6, pp.6897-6928, 2014.

[13] Du, P., Xia, J., Zhang, W., et al. "Multiple classifier system for remote sensing image classification: a review", Sensors, vol.12, pp.4764-4792, 2012.

[14] E. Kosmatopoulos, M. Polycarpou, "High-order neural network structures for identification of dynamical systems," IEEE Transactions on Neural Networks, vol. 6, no. 2, pp. 422-431, 1995.

\section{Creative Commons Attribution License 4.0 (Attribution 4.0 International, CC BY 4.0)}

This article is published under the terms of the Creative Commons Attribution License 4.0 https://creativecommons.org/licenses/by/4.0/deed.en_US 